\title{
STIEFEL-WHITNEY NUMBERS AND MAPS COBORDANT TO EMBEDDINGS
}

\author{
RICHARD L. W. BROWN 1
}

ABSTRACT. A necessary and sufficient condition is given for a continuous map between compact differentiable manifolds to be cobordant in the sense of Stong to an embedding. For the case of a map $f$ : $M^{n} \rightarrow S^{n+k}$ the condition reduces to the vanishing of all Stiefel-Whitney numbers of $M^{n}$ that involve $\bar{w}_{i}$ for $i \geq k$.

1. Introduction. A necessary condition for the existence of an embedding of a compact differentiable manifold $M^{n}$ in a euclidean space $R^{n+k}$ (or a sphere $S^{n+k}$ ) is the vanishing of the dual Stiefel-Whitney classes $\bar{w}_{i}\left(M^{n}\right) \in H^{i}\left(M^{n} ; Z / 2 Z\right)$ for $i \geq k$. This condition is far from sufficient. For example, if $M^{n}$ is a real projective $n$-space $P^{n}$ with $n=2^{s}-1(s \geq 4)$, then $\bar{w}_{i}\left(P^{n}\right)=0$ for all $i>0$, but $P^{n}$ does not embed in $R^{n+k}$ if $k<n / 4$. (See [1, p. 131].) However, one can still look for some statement involving embeddings that is implied by the condition $\bar{w}_{i}\left(M^{n}\right)=0$ for $i \geq k$. Because Stiefel-Whitney numbers form a complete system of invariants for certain cobordism theories one can expect a result involving cobordism, and in [2] we have shown that if $\bar{w}_{i}\left(M^{n}\right)=0$ for $i \geq k$ then $M^{n}$ is cobordant to a manifold $M_{1}^{n}$ that embeds in $S^{n+k}$ provided that $k$ is not much smaller than $n$. Equivalently, under the same conditions, any map $f: M^{n} \rightarrow S^{n+k}$ is bordant to an embedding $f_{1}: M_{1}^{n} \rightarrow S^{n+k}$.

In this paper we use the notion of cobordism of maps due to Stong and prove that the vanishing of all Stiefel- Whitney numbers of $M^{n}$ involving $\bar{w}_{i}\left(M^{n}\right)(i \geq k)$ is necessary and sufficient for a map $f: M^{n} \rightarrow S^{n+k}$ to be cobordant as a map to an embedding $f_{1}: M_{1}^{n} \rightarrow N_{1}^{n+k}$. In other words if you weak en the original embedding problem using cobordism of maps, then the whole story is told by the usual dual Stiefel-Whitney numbers.

Actually we solve the more general problem of determining when a map $f: M^{n} \rightarrow N^{n+k}$ is cobordant to an embedding $f_{1}: M_{1}^{n} \rightarrow N_{1}^{n+k}$. In the next

Received by the editors January 15, 1974.

AMS (MOS) subject classifications (1970). Primary 57D40, 57D75, 57D90.

Key words and phrases. Cobordism of maps, Stiefel-Whitney numbers, embeddings.

1 This work was partially supported by the National Research Council of Canada. 
section we develop some necessary conditions involving Stiefel-Whitney numbers of $f$, and in the third section we state the theorems and prove sufficiency of our conditions. Here the proof is based on a construction suggested to me by Stong. The last section is devoted to examples and remarks. Throughout we use homology and cohomology with $Z / 2 Z$ coefficients.

2. Stiefel-Whitney numbers of maps. Given a map $f: M^{n} \rightarrow N^{n+k}$, we have induced maps $f^{*}$ and $f_{*}$ in cohomology. Recall that if $x \in H^{i}\left(M^{n}\right)$ then $f_{*}(x)=D_{N} f_{*}(x \cap[M]) \in H^{i+k}\left(N^{n+k}\right)$, where $D_{N}$ denotes Poincaré duality for $N^{n+k}$, and where $f_{*}$ is also used to denote the induced homology map of $f$. Then Stong [5] shows that the cobordism class of $f$ is completely determined by the Stiefel- Whitney numbers of $f$, namely the numbers

$$
\left\langle w_{\omega}(N) \cdot f_{*} w_{\omega_{1}}(M) \cdot \ldots \cdot f_{*} w_{\omega_{r}}(M),[N]\right\rangle .
$$

Here $[N]$ denotes the fundamental ${ }_{i}$ homology class in $H^{n+k}(N), \omega=$ $\left(i_{1}, \cdots, i_{p}\right), w_{\omega}=w_{1}^{i_{1}} \cdot \ldots \cdot w_{p}{ }^{p},|\omega|=i_{1}+\cdots+i_{p}$, and $|\omega|+$ $\sum_{j=1}^{r}\left(\left|\omega_{j}\right|+k\right)=n+k$.

We find it convenient to rewrite those numbers with $r>0$ so as to have classes in $H^{*}(M)$ evaluated on $[M]$. Observe that

$$
\begin{gathered}
\left\langle a \cdot f_{*}(b) \cdot f_{*}(c),[N]\right\rangle=\left\langle a \cdot f_{*}(b), f_{*}(c) \cap[N]\right\rangle=\left\langle a \cdot f_{*}(b), f_{*}(c \cap[M])\right\rangle \\
=\left\langle f^{*}(a) \cdot f^{*} f_{*}(b), c \cap[M]\right\rangle=\left\langle f^{*}(a) \cdot f^{*} f_{*}(b) \cdot c,[M]\right\rangle .
\end{gathered}
$$

Thus the numbers of $f$ with $r>0$ take the form

$$
\left\langle f^{*} w_{\omega}(N) \cdot f^{*} f_{*} w_{\omega_{1}}(M) \cdot \ldots \cdot f^{*} f_{*} w_{\omega_{r-1}^{\prime}}(M) \cdot w_{\omega_{r}}(M),[M]\right\rangle .
$$

Now suppose that $f$ is an embedding with normal bundle $\nu$. Then $f^{*} f_{*}(a)=a \cdot w_{k}(\nu)$. (This is because $f_{*}$ has another interpretation, namely, $f_{*}=c^{*} \Phi$, where $c: N \rightarrow T(\nu)$ is the collapsing map of $N$ onto the Thom space of $\nu$, and $\Phi: H^{*}(M) \rightarrow H^{*+k}(T(\nu))$, is the Thom isomorphism. If $i$ : $M \rightarrow T(\nu)$ is the inclusion of the zero section then $f^{*} f_{*}(a)=f^{*} c^{*} \Phi(a)=i^{*} \Phi(a)$, and finally $i^{*} \Phi(a)=a \cdot w_{k}(\nu)$ by a basic property of the Thom isomorphism.) To see how $w_{k}(\nu)$ is determined by $f$, let $N$ be embedded in a euclidean space $R^{t}$ with normal bundle $\eta$. Then $\tau M^{n} \oplus \nu \oplus f^{-1} \eta$ is a trivial bundle, so $w(M) w(\nu) f^{*} \bar{w}(N)=1$, and hence $w(\nu)=\bar{w}(M) f^{*} w(N)$. Note that $w_{i}(\nu)=0$ if $i>k$ because $\nu$ is a $k$-dimensional bundle.

Definition. $\bar{w}(f)$ for any map $f: M^{n} \rightarrow N^{n+k}$ is defined by $\bar{w}(f)=$ $\bar{w}(M) f^{*} w(N)$. 
We now have a necessary condition for $f$ to be cobordant to an embedding, namely that $\bar{w}_{i}(f)$ should be zero in numbers if $i>k$, and that the numbers of the form (1) should be equal to the numbers of the form (2) below:

$$
\left\langle f^{*} w_{\omega}(N) \cdot w_{\omega_{1}}(M) \cdot \ldots \cdot w_{\omega_{r}}(M) \cdot\left(\bar{w}_{k}(f)\right)^{r-1},[M]\right\rangle .
$$

3. The main results.

Theorem. A map $f: M^{n} \rightarrow N^{n+k}(k>0)$ is cobordant to an embedding $f_{1}: M_{1}^{n} \rightarrow N_{1}^{n+k}$ if and only if the following conditions hold:

(i) All Stiefel-Whitney numbers of $f$ involving $\bar{w}_{i}(f)$ for $i>k$ are zero.

(ii) All Stiefel-Whitney numbers of $f$ as given by (1) are equal to the corresponding Stiefel-Whitney numbers as given by (2).

Proof. We have just shown that (i) and (ii) are necessary, so now let $f$ be a map that satisfies (i) and (ii). We wish to construct a cobordant embedding $f_{1}$. If $t$ is large, the map $(f, 0): M^{n} \rightarrow N^{n+k} \times \mathbf{R}^{t}$ is homotopic to an embedding with normal bundle $\eta$ classified by a map $\bar{\eta}: M^{n} \rightarrow B O$. Because $\tau M \oplus \eta \simeq f^{-1} \tau N \oplus t \epsilon$, we see that $w(\eta)=\bar{w}(M) \cdot f^{*} w(N)=\bar{w}(f)$. It follows that the Stiefel-Whitney numbers of the map $\eta$ which are used to determine the bordism class of this map are a subset of the Stiefel-Whitney numbers of the map $f$, and the condition (i) of the hypotheses implies that all StiefelWhitney numbers of $\bar{\eta}$ involving $w_{i}(\eta)$ for $i>k$ are zero. Hence $\bar{\eta}$ is bordant to a map that factors through $B O(k)$, say $\bar{\eta}_{1}: M_{1}^{n} \rightarrow B O(k) \subset B O$ with associated bundle $\eta_{1}$ over $M_{1}^{n}$. (See $\left[3,17.3\right.$, p. 48].) Let $S\left(\eta_{1} \oplus 1\right)$ denote the sphere bundle of $\eta_{1} \oplus 1$ over $M_{1}$, let $N_{1}^{n+k}=S\left(\eta_{1} \oplus 1\right) \cup N^{n+k}$, and let $f_{1}: M_{1}^{n} \rightarrow N_{1}^{n+k}$ be the inclusion of the cross-section determined by the trivial line bundle. (I wish to thank R. E. Stong for showing me this construction in the case where $N^{n+k}=S^{n+k}$.) Then $f_{1}$ is a differentiable embedding, and it remains to show that $f$ and $f_{1}$ are cobordant. For this purpose we compute the Stiefel-Whitney numbers of $f_{1}$ and compare them with those of $f$.

Because of the section, $H^{*}\left(M_{1}^{n}\right)$ is a direct summand of $H^{i k}\left(S\left(\eta_{1} \oplus 1\right)\right)$ so the Serre spectral sequence for $H^{*}\left(S\left(\eta_{1} \oplus 1\right)\right)$ collapses and $H^{*}\left(S\left(\eta_{1} \oplus 1\right)\right)$ is isomorphic to $H^{*}\left(M_{1}^{n}\right) \oplus H^{*}\left(S^{k}\right)$. If $p: S_{1}=S\left(\eta_{1} \oplus 1\right) \rightarrow M_{1}$ is the projection, then $\tau S_{1}=p^{-1} \tau M_{1} \oplus \phi$, where $\phi$ is the bundle along the fibres. Thus $f_{1}^{-1} \tau N_{1}=\tau M_{1} \oplus f_{1}^{-1} \phi=\tau M_{1} \oplus \eta_{1}$, and we obtain the equations

$$
f_{1}^{*} w\left(N_{1}\right)=w\left(M_{1}\right) \cdot w\left(\eta_{1}\right) \text { and } \quad w\left(\eta_{1}\right)=\bar{w}\left(M_{1}\right) f^{*} w\left(N_{1}\right)=\bar{w}\left(f_{1}\right) .
$$

Now we are ready to compare numbers. First the numbers of $f$ and $f_{1}$ with $r=0$ both equal the numbers of $N$ because $S\left(\eta_{1} \oplus 1\right)$ is a boundary. 
If $r>0$, the numbers of $f$ and $f_{1}$ of the form (1) reduce to those of the form (2) by hypothesis (ii) for $f$ and by construction (i.e., $f_{1}$ is an embedding) for $f_{1}$. Now we use the fact that $f^{*} w(N)=w(M) \cdot \bar{w}(f)=w(M) w(\eta)$ and the analogous fact for $f_{1}$ to rewrite the number of the form (2) into the form $\left\langle w_{\omega}(M) w_{\omega^{\prime}}(\eta),[M]\right\rangle$ with an analogous expression for $f_{1}$. But now we are looking at Stiefel-Whitney numbers of $\bar{\eta}$ and of $\bar{\eta}_{1}$ and these are equal because $\bar{\eta}$ and $\bar{\eta}_{1}$ are bordant maps. This completes the proof.

Corollary. A map $M^{n} \rightarrow S^{n+k}(k>0)$ is cobordant to an embedding $f_{1}$ : $M^{n} \rightarrow N_{1}^{n+k}$ if and only if all Stiefel-Whitney numbers of $M^{n}$ involving $\bar{w}_{i}\left(M^{n}\right)(i \geq k)$ are zero. (In interpreting the statement of the Corollary it helps to note that all maps $f: M^{n} \rightarrow S^{n+k}(k>0)$ are cobordant.)

Proof. Taking $N^{n+k}=S^{n+k}$ in the Theorem we find that $w\left(S^{n+k}\right)=1$ and that $f^{*} f_{*}(x)=0$ for all $x \in H^{*}(M)$. Thus condition (i) is equivalent to saying that all Stiefel-Whitney numbers involving $\bar{w}_{i}\left(M^{n}\right)(i>k)$ should vanish, and condition (ii) is then equivalent to saying that all numbers involving $\bar{w}_{k}\left(M^{n}\right)$ should vanish.

4. Applications and examples. If we apply the Corollary to a product we obtain the following result.

Proposition 1. If maps $M^{m} \rightarrow S^{m+p}, N^{n} \rightarrow S^{n+q}$ are both cobordant to embeddings, then any map $M^{m} \times N^{n} \rightarrow S^{m+n+p+q-1}$ is cobordant to an em. bedding. In other words, products always embed better (modulo map corbordism) than the product embedding of the factors. We assume $p>0, q>0$.

Proof. This follows from the Corollary because the top nonzero class (in numbers) of $M^{m} \times N^{n}$ is $\bar{w}_{p+q-2}\left(M^{m} \times N^{n}\right)=\bar{w}_{p-1}\left(M^{m}\right) \cdot \bar{w}_{q-1}\left(N^{n}\right)$.

Remark. One can ask whether the proposition is true without the "modulo map cobordism" clause. In many cases it does hold. For let $d(X)$ denote the difference between the best euclidean immersion and best euclidean embedding of the manifold $X$. If $d\left(M^{m}\right)>0$ and $m \leq n+p+q-2$, then we can embed $M^{m} \times N^{n}$ in $R^{m+n+p+q-1}$ given embeddings of $M^{m}$ in $R^{m+p}$ and $N^{n}$ in $R^{n+q}$. (See [4, p. 319].) But by [4, pp. 320, 321] the product embedding of $\left(C P^{2}\right)^{2}$ is best possible. However this manifold is cobordant to $\left(R P^{2}\right)^{4}$ whose product embedding is not best. Hence "modulo map cobordism" cannot be deleted but might possibly be improved to "modulo bordism".

Now consider the case $M^{n}=P^{n}$, a real projective $n$-space. Recall that if $\alpha$ generates $H^{1}\left(P^{n}\right)$ then $H^{*}\left(P^{n}\right)=(Z / 2 Z)[\alpha] /\left(\alpha^{n+1}\right)$ and $w\left(P^{n}\right)=$ 
$(1+\alpha)^{n+1}$. If $n$ is odd then all Stiefel-Whitney numbers of $P^{n}$ are zero and a map $P^{n} \rightarrow S^{n+k}(k>0)$ is cobordant to the obvious embedding $S^{n} \subset S^{n+k}$. So let $n$ be even and write $n=2^{a}+b$ with $0 \leq b<2^{a}$.

Proposition 2. A map $f: P^{n} \rightarrow S^{n+k}$ ( $n$ even, $k>0$ ) is cobordant to an embedding if and only if $k \geq n-2 b$.

Proof. $\bar{w}\left(P^{n}\right)=(1+a)^{-n-1}=(1+a)^{-2^{a+1}}(1+a)^{2^{a}-b-1}=$ $\left(1+a^{2^{a+1}}\right)^{-1}(1+a)^{2^{a}-b-1}=(1+a)^{2^{a}-b-1}$. Let $p=2^{a}-b=n-2 b$. Then $\bar{w}_{i}\left(P^{n}\right)=0$ if $i \geq p$ but the Stief el-Whitney number $w_{1}^{n-p+1} \bar{w}_{p-1} \neq 0$.

Remark. The extreme cases are $n=2^{a}$ and $n=2^{a+1}-2$. In the first case we get $k \geq n$ and this shows that a high codimension may be needed even for embeddings modulo map cobordism. In the second case we get $k \geq 2$. An example of a codimension 2 embedding $f_{1}$ cobordant to $f$ may be constructed as follows. Let $M_{1}^{n}=P^{n}$, let $N_{1}^{n+2}=P^{n+1} \times S^{1}$, and let $f_{1}$ be the inclusion of $P^{n}$ into $P^{n+1} \times\{1\}$. Then the normal bundle of $f_{1}$ admits a section, so $\bar{w}_{2}\left(f_{1}\right)=0$, and hence $f_{1}^{*} f_{1 *}(x)=0$ for all $x$. Also $w\left(N_{1}^{n+2}\right)=$ 1. Thus the Stiefel-Whitney numbers of $f_{1}$ reduce to those of $P^{n}$ and the same is true for the Stiefel-Whitney numbers of $f$.

Proposition 3. Let $f: P^{n} \rightarrow P^{n+k}(k>0)$ be a map. If $f^{*}(\alpha) \neq 0$ then $f$ is cobordant to the inclusion $P^{n} \subset P^{n+k}$. If $f^{*}(\alpha)=0$ then $f$ is cobordant to an embedding if and only if $n$ is odd or $k \geq n-2 b$ where $n=2^{a}+b$ as above.

Proof. If $f^{*}(\alpha)=\alpha$ (with the obvious abuse of notation) then $f$ has the same Stiefel-Whitney numbers as the inclusion $P^{n} \subset P^{n+k}$. If $f^{*}(\alpha)=0$ then $f^{*} f_{*}(x)=0$ for all $x$, and $f^{*} w\left(P^{n+k}\right)=1$. Thus $f$ has the same StiefelWhitney numbers as a map $P^{n} \rightarrow S^{n+k}$ and Proposition 2 applies.

Finally we mention the homotopy theoretic interpretation of our results. Stong [5] has shown that cobordism classes of maps from $n$-manifolds to $(n+k)$-manifolds are in 1-1 correspondence with the bordism group $\Re_{n+k}\left(\Omega^{s} M O(k+s)\right), s$ large. On the other hand, $\Re_{n+k}(M O(k))$ represents cobordism classes of embeddings of codimension $k$. The obvious map $B O(k) \rightarrow$ $B O(k+s)$ yields a map $\Sigma^{s} M O(k) \rightarrow M O(k+s)$ and hence a map $\Psi: M O(k) \rightarrow$ $\Omega^{s} M O(k+s)$. The induced homomorphism $\Re_{*}(\Psi)$ on bordism is injective. (For the Stiefel-Whitney numbers of a map $\phi: N^{n+k} \rightarrow M O(k)$ can be written in terms of the associated embedding $f: M^{n} \rightarrow N^{n+k}$ and take the form (2) of $\$ 2$. They are included among the Stiefel-Whitney numbers of $f$ considered now as a map.) We have described the image of $\Re_{*}(\Psi)$ in terms of comput- 
able invariants. There is another problem whose solution must also be given by Stiefel-Whitney numbers, namely finding which bordism classes of maps $f: M^{n} \rightarrow N^{n+k}$ can be represented by an embedding $f_{1}: M_{1}^{n} \rightarrow N^{n+k}$. In terms of homotopy theory we are trying to describe the image of the set of homotopy classes $\left[N^{n+k}, M O(k)\right]$ in the group $\left[N^{n+k}, \Omega^{s} M O(k+s)\right]=$ $\Re^{k}\left(N^{n+k}\right)=\Re_{n}\left(N^{n+k}\right)$. (See [3, p. 37].) The appropriate Stiefel-Whitney numbers of $f$ have the form $\left\langle w_{\omega}(M) f^{*}(x),[M]\right\rangle$, where $x \in H^{*}(N)$ need not be a characteristic class of $N$. In the case where $N^{n+k}=S^{n+k}$, these numbers reduce to the numbers of $M^{n}$ and it would be interesting to know the answer here and to see whether new nonembedding theorems could be deduced using Stiefel-Whitney classes.

\section{REFERENCES}

1. M. F. Atiyah, Immersions and embeddings of manifolds, Topology 1 (1962), 125-132. MR 26 \#3080.

2. R. L. W. Brown, Immersions and embeddings up to cobordism, Canad. J. Math. 13 (1971), 1102-1115. MR 45 \#6017.

3. P. E. Conner and E. E. Floyd, Differentiable periodic maps, Ergebnisse der Mathematik und ihrer Grenzgebiete, N. F., Band 33, Academic Press, New York; Springer-Verlag, Berlin, 1964. MR 31 \#750.

4. B. J. Sanderson and R. L. E. Schwarzenberger, Non-immersion theorems for differentiable manifolds, Proc. Cambridge Philos. Soc. 59 (1963), 319-322. MR $26 \# 5589$.

5. R. E. Stong, Cobordism of maps, Topology 5 (1966), 245-258. MR 33 \#4945.

DEPARTMENT OF MATHEMATICS, YORK UNIVERSITY, TORONTO, ONTARIO, CANADA 